\title{
Once bitten, twice shy: Experienced regret and non-adaptive choice switching
}

Francesco Marcatto, Anna Cosulich, Donatella Ferrante

When a good decision leads to a bad outcome, the experience of regret can bias

subsequent choices: People are less likely to select the regret-producing alternative a second time, even when it is still objectively the best alternative (non-adaptive choice switching). The first study presented herein showed that nearly half of participants experiencing regret rejected a previous alternative they had recognized as the best one, and chose a non-optimal alternative instead. The second study investigated the mechanism underlying this bias, and results supported the hypothesis that this nonadaptive choice switching is caused by inhibition of the previous decision (direct effect of experienced regret), rather than by increased sensitivity to anticipated regret in subsequent choices (indirect effect of experienced regret mediated by anticipated regret). 
2 TITLE: Once bitten, twice shy: Experienced regret and non-adaptive choice switching 3

4 SHORT TITLE: Regret and non-adaptive choice switching 5

6

7

8 AUTHORS:

9 Francesco Marcatto, Department of Life Sciences, University of Trieste, Italy

10 Anna Cosulich, Department of Life Sciences, University of Trieste, Italy

11 Donatella Ferrante, Department of Life Sciences, University of Trieste, Italy

12

13

14

15

16

17 Address correspondence to: Francesco Marcatto, Department of of Life Sciences, Via Weiss 21, 34128

18 Trieste, Italy;

19 E-mail: fmarcatto@units.it

20 Phone: +393401575454 


\section{INTRODUCTION}

23 Regret is an emotion with strong cognitive roots, based on a comparison between "what is" and "what

24 might have been", that is, between the outcome we actually obtained and a better outcome we would

25 have obtained, had we chosen differently (van Dijk \& Zeelenberg, 2005). Regret happens, it is

26 unavoidable. Indeed, how many of us have not wanted to just kick ourselves, at least once, for

27 something we did (or did not do)? When we look back and see that things would have turned out better

28 had we made a different choice, we frequently experience this unpleasant emotion (Marcatto \&

29 Ferrante, 2012). In order to feel regret, we do not need to actually observe that we could have obtained

30 a better outcome, it is sufficient to imagine it (Kahneman, 1995; Bar-Hillel \& Neter, 1996). In

31 decision-making research, regret is usually differentiated from disappointment: Both are negative

32 emotions that arise from counterfactual comparison, but while regret results from a comparison

33 between an actual outcome and a better outcome that might have occurred had we made a different

34 choice, disappointment stems from comparing an obtained outcome with a better outcome that might

35 have occurred had we made the same choice. This distinction is particularly relevant because these

36 emotions have distinguishable behavioral consequences for decision making (see, e.g., van Dijk \&

37 Zeelenberg, 2002; Zeelenberg et al., 2000).Why do we feel regret? Isn't it useless to cry over spilled

38 milk? It is generally assumed that although painful, regret is functional because it helps us make better

39 decisions. According to the Regret Theory (Bell, 1982; Loomes \& Sugden, 1982), we can anticipate

40 the possible emotional consequences of our choices before we make them, and use these anticipated

41 emotions to guide our choices (Janis \& Mann, 1977; Mellers, Schwartz \& Ritov, 1999). Thus, fear of

42 possible future regret let us usually avoid risky behaviors and painful experiences (e..g., Zeelenberg,

43 1999a; Wright \& Ayton, 2005). Moreover, experienced regret (or retrospective regret) may help us to

44 prevent similar mistakes in the future, since it makes the mistakes more painful (Zeelenberg, 1999b;

45 Saffrey, Summerville \& Roese, 2008). According to a popular quote, "Regret is insight that comes a 
46 day too late". In fact, when we think that we should have behaved differently, we learn something, for

47 example that we should avoid the restaurant that served bad food or that drinking too much has its

48 downsides. Thus, being able to look back on and evaluate our past choices allows us to modify future

49 behavior and presumably make better decisions.

51 Regret and adaptive choices

52 The idea of an adaptive role of the cognitive-based emotion of regret is supported by many research

53 findings. Many studies have shown that people are regret averse: They spontaneously anticipate future

54 regret and behave in ways that minimize the possibility of experiencing this negative emotion, for

55 example by avoiding risky choices (Richard, van der Pligt \& de Vries, 1996; Wright \& Ayton, 2005),

56 or by avoiding feedback about foregone alternatives (Larrick \& Boles, 1995; Ritov, 1996; Zeelenberg

57 et al., 1996; Hoelzl \& Loewenstein, 2005; van de Ven \& Zeelenberg, 2011).

58 Neuropsychological studies showed that patients with selective lesions to the orbitofrontal cortex, an

59 area associated with the processing of counterfactual comparisons, perform worse than controls in

60 repeated gambling tasks, since they cannot learn from their prior emotional experiences (Camille et al.,

61 2004; Coricelli, Dolan \& Sirigu, 2007).

62 Recently O'Connor, McCormack \& Feeney (2014) investigated the development of regret in children,

63 and found that when children begin to experience regret (between the ages of 5 and 7 years), the

64 quality of their subsequent decision making improves. The development of regret allows children to

65 learn from their previous choices and thus to make better choices when faced with the same situation

66 again, a behavior that the authors have termed adaptive choice switching.

67 A similar regret-based learning process, called regret matching, has also been implemented in

68 algorithms used in game theory. According to this procedure, in repeated games the player changes his

69 current strategy for a foregone alternative that would have given a higher payoff in the past (Hart \& 
70 Mas-Colell, 2000; Hart, 2005; Marchiori \& Warglien, 2008). Regret matching is a simple adaptive

71 procedure, based only on comparison of the realized payoff and the foregone payoffs, leading in the

72 long run to a sophisticated solution of the game (correlated equilibrium) (Coricelli, Dolan \& Sirigu, 73 2007).

75 Non-adaptive choice switching

76 Is regret-induced choice switching always adaptive? What happens, for example, when people

77 experience regret following an optimal decision, and are faced with the same choice again? When

78 regret is the unfortunate consequence of a good decision, or when previous outcomes are not related to

79 subsequent choices, switching choices blindly could lead to biased decisions.

80 Do people actually make "bad decisions" as a consequence of regret? Some literature findings suggest

81 that this can happen. Preliminary evidence was observed in a study conducted by Zeelenberg \& Beattie

82 (1997). In one of their experiments, participants played two rounds of Ultimatum Game as offerers.

83 After the first round, they were told that their offer was either 2 Guilders or 10 Guilders higher than the

84 responder's minimal acceptable offer. Participants discovering they had offered 10 Guilders too much

85

86

87 experienced regret and lowered their offer in the subsequent round thereby, even if they knew that they were playing with a new responder, whose minimal acceptable offer could have easily differed from the previous responder's offer. Similar results were found also in neuroimaging studies conducted by Büchel, Brassen and colleagues (Büchel et al., 2011; Brassen et al., 2012) aimed at investigate the neural mechanisms for how missed opportunities influence future choices. Their results showed that, in a sequential risk taking task, following a missed opportunity (e.g., you would have obtained a higher gain, if you had risked more) participants increased the risk taken in the next round, despite the fact that consecutive rounds were explicitly independent, and this behavior was paralleled by signal changes in the ventral striatum, a neural structure involved in regret processing. 
94 Ratner \& Herbst (2005) found another evidence for this effect. Their experiments were based on a

95 scenario methodology requiring participants to choose between two brokers, one of whom was

96 described as having a better success rate than the other. Obviously, most participants chose the broker

97 with the better success rate. Half of them were then informed that the selected broker had succeeded,

98 the others were told that their broker had failed, and all of them were then asked to imagine which of

99 the two brokers they would choose the next time. Results showed that participants whose broker had

100 failed regretted their decision and reported a lower intention to select the same broker again, even if she

101 still had the best success rate.

102

\section{Choice switching: Experienced vs. anticipated regret}

104 Since most studies investigating the behavioral consequences of regret have adopted repeated gambling 105 tasks involving many trials, it is usually difficult to disentangle the effect of anticipated from 106 experienced regret. Indeed, O'Connor, McCormack \& Feeney (2014) hypothesized two mechanisms

107 that might explain the choice switching behavior. The first one is a simple process, consisting in

108 remembering that a particular option yielded a poor result in the past, thus avoiding it when faced with

109 the same choice again (direct effect of experienced regret, henceforth direct effect). According to the

110 alternative explanation, the effect of experienced regret on subsequent choices is mediated by

111 anticipated regret: A recent experience of regret could prime the anticipation of regret in the following

112 choices, leading to increased regret aversion (indirect effect of experienced regret mediated by

113 anticipated regret, henceforth indirect effect). The finding that the same neural circuitry mediates the

114 experience of regret and its anticipation, emerged in brain imaging studies (Coricelli et al., 2005),

115 provide support for the latter hypothesis. On the other hand, in the O'Connor, McCormack \& Feeney

116 (2014) studies on regret in children provided evidence for the direct effect mechanism: The authors

117 found a choice switching behavior in children who have already developed the ability to experience 
118 regret, but were still incapable of anticipating it, thus showing that experienced regret can affect a

119 future choice independent of anticipated regret.

\section{The current studies}

122 On the basis of the aforementioned considerations, the present studies were aimed at (i) providing

123 further evidence of a non-adaptive choice switching behavior using real choices (Study 1), and at (ii)

124 trying to shed light in the process that underpin this bias, by using a task in which the two hypothesized

125 mechanisms (direct effect vs. indirect effect) would lead to different behaviors (Study 2).

126 Both studies adopted the choice switching paradigm (O’Connor, McCormack \& Feeney, 2014).

127 Specifically, a first choice was followed by feedback about the respective outcomes of the chosen and

128 non-chosen alternatives. The aim was to induce either regret (i.e., you obtained a bad outcome, and the

129 non-chosen alternative would have been better) or disappointment (i.e., you obtained a bad outcome

130 and even the non-chosen alternative would have produced the same outcome) ${ }^{1}$. Afterwards, a second

131 choice was presented, either similar (Study 1) or different (Study 2) from the previous. Unlike previous

132 studies on experienced regret, this experimental design made it possible to disentangle the specific

133 effect of regret from a more general effect caused by disappointment for the negative outcome. It was

134 hypothesized that, if regret influences subsequent decisions, participants who receive the regret

135 feedback should switch from a good decision to a non-optimal one in a subsequent choice, as opposed

136 to participants receiving the disappointment feedback.

\section{STUDY 1}

\footnotetext{
${ }^{1}$ Disappointment is often induced by showing the bad outcome only, participants do not actually see what the counterfactual outcome would have been (e.g., Mellers, Schwartz \& Ritov, 1999). We decided to give participants a feedback also in disappointment conditions, to avoid the possibility that participants spontaneously produced regretinducing counterfactual thoughts (e.g., "if only I had chosen differently, I would have obtained a better outcome").
} 


\section{Method}

\section{Participants and design}

141 A total of 105 students $(70$ women and 35 men; mean age $=22.2$ years, $S D=3.25)$ from Trieste

142 University voluntarily participated in the study and were randomly assigned to either the regret- or

143 disappointment condition. Participants were tested individually and were advised about the data

144 retained and that anonymity was fully ensured, no sensitive data were collected.

\section{Procedure}

147 Participants played two rounds of a simplified computer-version of Blackjack against the computer-

148 played dealer. To increase overall motivation and involvement in the game, each round had a 5-Euro

149 prize. Before beginning the first round, participants received instructions on Blackjack rules.

150 Participants started playing by turning their initial two cards face up, and then had to choose whether to

151 take more cards or to stop, by pressing two buttons labeled "draw" and "stop", respectively. Both

152 dealer's initial cards were face down, to prevent participants from using casino Blackjack strategies

153 like basic (for a review on the psychology of playing Blackjack, see Keren \& Wagenaar, 1985). The

154 game was rigged so that in the first round all participants received the same cards (a " 2 ", a "6" and a

155 picture card), thus achieving the score of 18 . We assumed that participants would have recognized that

156 stopping was the optimal decision after having achieved the score of 18 . In any event, participants who

157 decided to draw another card would receive an " 8 ", losing the game thereby. The dealer played after

158 the participants and won by scoring 19 (with a picture card and a "9"); all participants therefore lost.

159 Participants who decided to stop at " 18 " then received a feedback about what would had happened, had

160 they chose to take another card ${ }^{2}$. Participants in the regret condition were shown that they would have

\footnotetext{
2 Participants choosing to take another card at " 18 " were not assigned to the experimental conditions and did not play the second round.
} 
161 drawn a "2", winning thereby with a score of 20 (regret feedback), while participants in the

162 disappointment condition were shown that they would have drawn an "8", and that they therefore

163 would have lost anyway (disappointment feedback). Participants' emotional reactions were assessed

164 with the Regret and Disappointment Scale (Marcatto \& Ferrante, 2008), a six-item scale measuring the

165 intensity of negative affect resulting from the bad outcome, regret and disappointment.

166 In the second round, participants achieved the score of 18 again, but this time with different cards (an

167 " 8 " and a picture card), and they once more had to choose to either take another card or to stop. We

168 therefore expected that, if previously experienced regret can induce non-adaptive choice switching,

169 participants in the regret condition would less likely choose the optimal alternative ("to stop") than the

170 participants in the disappointment condition would.

171

172 Results

173 In the first round of Blackjack, most participants (90 out of 105, 86\%) decided to stop after scoring 18.

174 As hypothesized, participants correctly recognized that stopping was the optimal decision. The data

175 from the 15 participants who decided to take another card were excluded from the analyses reported in 176 the next section.

\section{Emotional reaction ratings}

179 As reported in Table 1, the two conditions induced similar levels of negative affect $(t=.20, d f=88, p$

$180=.84, d=0.04)$, but different types of specific emotions: The regret score was higher in the regret

181 condition than in the disappointment condition $(t=7.80, d f=88, p<.001, d=1.65)$, and the

182 disappointment score was higher in the disappointment condition than in the regret condition $(t=4.98$,

$183 d f=88, p<.001, d=1.05)$. Due to the key role of chance in Blackjack, high disappointment scores

184 were expected for both conditions. 
185 Second Blackjack round choices

186 In the second round, $44 \%$ (20 out of 45 ) of the regret condition participants decided to take another

187 card after having scored 18 , whereas only $24 \%$ (11 out 45$)$ of the disappointment condition participants

188 decided to do so $\left(\chi_{(1,90)}^{2}=3.99, p<.05\right.$, Cramér's $\left.V=.210\right)$.

189 A logistic regression was then conducted to assess whether the participants' choices in the second

190 round could be significantly predicted by the following variables: Type of feedback (regret or

191 disappointment), negative affect, regret score and disappointment score. Since predictors were

192 expected to be highly correlated, a stepwise method with backward elimination was used. Regret score

193 turned out to be the only significant predictor, with higher regret scores being associated with an

194 increased likelihood of deciding to take another card $(B=.45 \text {, Wald }=7.89, p<.01)^{3}$.

195

\section{Discussion}

197 Nearly half of the regret condition participants, correctly stopping at the score of 18 in the first round,

198 switched their choice in the second round by deciding to take a further card, whereas most

199 disappointment condition participants once more decided for the optimal alternative.

200 Our results therefore showed that choice switching can happen also following the unfortunate failure of

201 an optimal choice (non-adaptive choice switching). Most important, choice switching turned out to be

202 associated with the intensity of experienced regret, as showed in the regression analysis. Thus, we

203 could expect an even stronger effect in real-life situations with a greater level of involvement.

205 STUDY 2

206 Method

${ }^{3}$ Other predictors excluded from the final regression model: Type of feedback (Wald $=.03, p=.87$ ), negative affect ( Wald $=1.52, p=.22)$, disappointment score (Wald $=.20, p=.65)$. 
Participants and design

208 A total of 80 students (49 women and 31 men; mean age $=22.9$ years, $S D=2.69$ ) from Trieste

209 University, who did not previously take part in Study 1, participated voluntarily. They were randomly

210 assigned to three experimental conditions: control condition $(\mathrm{N}=25)$, regret condition $(\mathrm{N}=28)$ and

211 disappointment condition $(\mathrm{N}=27)$. Participants were tested individually and were advised about the

212 data retained and that anonymity was fully ensured, no sensitive data were collected.

\section{Procedure}

215 As in Study 1, participants played a first round of Blackjack with a 5-Euro prize and received a

216 feedback (regret or disappointment) about the previous choice (to take another card or to stop at the

217 score of 18). Afterwards, they played a round of Red \& Black, a simple computerized card game

218 similar to the one proposed originally by Slovic (1966) and used more recently in other studies (e.g.,

219 Fernandez-Duque \& Wifall, 2007). In this game participants were presented 10 cards lying face down

220 into two rows on the computer screen, they were told there were nine red queens and a single black

221 queen. Their task consisted in deciding how many and which cards to turn face up, by mouse-clicking

222 on the back of the cards, one by one: Every red queen increased their jackpot by 0.50 Euros, but the

223 black queen (the "disaster" card) resulted in a total loss and ended the game. Participants could choose

224 to finish playing and collect their jackpot at any moment by pressing the "stop" button. To obviate the

225 possibility of participants finding the black queen too early on, the game was rigged to have the black

226 queen appear only if participants decided to continue the game until the eighth card.

227 Participants in the control condition played only Red \& Black, without the previous Blackjack round.

228 Red \& Black participants' behavior was diagnostic of the underlying regret mechanism. Differently

229 from previous versions of this game used to investigate the effects of missed opportunities (e.g.,

230 Büchel et al., 2011; Brassen et al., 2012), our participants were not forced to turn the cards 
231 sequentially, instead they were free to choose the cards in whatever order they preferred. Moreover, the

232 position of the "disaster" card was not shown after participants stopped. This means that in our version

233 of the game our participants should not feel regret for having missed the opportunity to gain more (e.g.,

234 counterfactuals like "I could have turned two more cards safely" are very unlikely). Thus, in this game

235 the regret-minimizing behavior consists in stopping early, to avoid receiving the "disaster" card. If

236 regret influences subsequent choices by increasing future regret aversion (indirect effect), participants

237 in the regret condition should have engaged in regret minimizing behavior, by deciding to stop after

238 turning over fewer cards than participants in the disappointment and control conditions. Alternatively,

239 if regret influences subsequent choices by inhibiting a previously selected option (direct effect), regret

240 condition participants were expected to avoid the decision causing regret in the previous Blackjack

241 round (i.e., stopping), to the extent that that they would have turned over more cards than participants

242 in the other conditions.

\section{Results}

245 Most participants in the first round of Blackjack (50 out of 55, 91\%) decided to stop after scoring 18.

246 Thus, as in Study 1, participants correctly recognized this as the optimal decision. Data from the 5

247 participants who decided to continue and take another card were excluded from the analyses reported

248 in the next section.

249 Choices in Red \& Black

250 Table 2 shows the mean and the median number of cards turned over in Red \& Black for each of the

251 three conditions. Data were analyzed using non-parametric tests, since regret condition's data were

252 highly skewed. A Kruskal-Wallis test yielded a significant difference among the three conditions

$253\left(\chi_{(2,75)}^{2}=7.83, p=.02\right)$. Planned comparison revealed that participants in the regret condition turned

254 over significantly more cards than participants in the disappointment condition (Mann-Whitney $Z=$ 
$2552.30, p=.02$ ) and participants in the control condition (Mann-Whitney $Z=2.53, p=.01$ ) did, with no

256 significant difference, however, between disappointment and control condition participants (Mann-

257 Whitney $Z=0.41, p=.68)$. Moreover, more than half of the participants in the regret condition (14 out

258 of $25,56 \%$ ) lost the game by turning over cards until arriving at the "disaster" card (card nr. 8),

259 whereas only $20 \%$ of the participants in the other two conditions ( 5 out of 25 in both the control and

260 disappointment conditions) continued until the eighth card.

\section{Discussion}

263 Without a prior experience of regret (control and disappointment conditions), in the Red \& Black game 264 participants tended to stop after turning over approximately 6 cards; this behavior was not far from the 265 game's actual optimal strategy (maximum expected value at 5 cards). Participants with a previous 266 experience of regret (regret condition), however, decided to turn over more cards, and $56 \%$ of these 267 lost the game, by turning over eight cards.

268 This result has multiple implications. Firstly, a non-adaptive choice switching behavior induced by 269 regret was once again confirmed. Secondly, this effect was not found to be domain-specific, thus regret 270 experienced after a decision task can influence people's behavior in a different subsequent task (see 271 also Creyer \& Ross, 1999; Raeva, Mittone \& Schwarzbach, 2010). Thirdly and most importantly, these 272 findings support the hypothesis of a direct effect of experienced regret on subsequent choices: Regret 273 leads to the rejection of the decision that previously resulted in a bad outcome. Thus, people are less 274 likely to make this decision again in a subsequent choice, and this could happen regardless of its 275 likelihood of success.

\section{CONCLUSIONS}


278 Research has demonstrated that people try to avoid future experiences of regret by opting for behavior

279 and choices that minimize the possibility of feeling this negative emotion (see, e.g., Simonson, 1992;

280 Beattie et al., 1994). Yet, things do not always go as planned, and regret is unfortunately a common

281 and somewhat painful experience that can influence future behavior: People are less likely to once

282 more opt for a decision that previously led to regret, and will conversely select other alternatives. This

283 behavior has been termed adaptive choice switching, since it is usually functional, allowing people to

284 learn from their previous mistakes and decreasing the probability of repeating negative outcomes

285 (Baumeister et al., 2007; Zeelenberg \& Pieters, 2007; O’Connor, McCormack \& Feeney, 2014). Yet, as

286 demonstrated by the studies reported herein, after a regret experience people may avoid the previously

287 made decision, even when it is still better than the other alternatives. In Study 1, participants played

288 two rounds of Blackjack and in the second round almost half of them switched away from the good

289 decision and selected the non-optimal decision as a consequence of having received a regret feedback

290 at the end of the previous round. In Study 2, the same pattern emerged even when the second round of

291 Blackjack was substituted with a different game, thus revealing a carry-over effect of experienced

292 regret also on a subsequent situation not directly related to the one that produced regret. Moreover,

293 Study 2 shed light on the mechanism underlying this bias by using a game in which the two

294 hypothesized mechanisms (direct effect vs. indirect effect) would have led to different behaviors.

295 Results supported the hypothesis that regret influences subsequent choices by inhibiting the previous

296 decision (direct effect): If this option becomes available again in a subsequent decision task, it is less

297 likely to be chosen, regardless of its intrinsic value. We conversely found no evidence supporting the

298 hypothesis that the effect of regret consists in priming the anticipation of regret in subsequent choices

299 (indirect effect). This result is consistent with recent work conducted by Raeva, van Dijk \& Zeelenberg

300 (2011), who found no evidence of increased regret anticipation after a recent experience of regret, and 
301 by O'Connor, McCormack \& Feeney (2014), who found behavioral consequences of regret in children 302 incapable of anticipating regret.

303 Overall, it appears that the experience of regret induces a strong tendency to avoid a decision that had

304 previously led to regret, and that this can occur even when it remains objectively the best one available.

305 Understanding the reasons people behave this way, abandoning a good decision for a non-optimal one

306 after an experience of regret, is theoretically challenging. We argue that this effect is a bias resulting

307 from the use of what we might call the "regret heuristic", a simple strategy that consists in basing

308 actual choice on the outcome of the previous choice. This strategy could be viewed as a particular

309 instance of the affect heuristic (Finucane et al., 2000), in which a previous decision is tagged with a

310 negative affect after having learned, through explicit feedback (as in the present studies), or after

311 having imagined (by counterfactual thinking), that a different decision would have been better. This

312 regret-mediated information is highly available, thus the previous outcome can be used as a heuristic

313 attribute in place of the target attribute, which in this instance is the option's likelihood of success

314 (Kahneman \& Frederick, 2002; Kahneman, 2003).

315 The findings presented in this paper highlight the possibility of the biased consequences of regret and

316 suggest a new account for these effects under a broader perspective. We do not claim that this paper

317 represents an exhaustive study, further research is obviously needed to explore the behavioral

318 consequences of experienced regret and the consistency of the regret heuristic hypothesis. 


\section{REFERENCES}

320 Bar-Hillel M, Neter E. 1996. Why are people reluctant to exchange lottery tickets? Journal of 321 personality and social psychology 70(1): 17-27.

322 Baumeister RF, Vohs KD, DeWall NC, Zhang L. 2007. How Emotion Shapes Behavior: Feedback, 323 Anticipation, and Reflection, Rather Than Direct Causation. Personality and Social Psychology Review 324 11(2): 167-203.

325 Beattie J, Baron J, Hershey JC, Spranca MD. 1994. Psychological determinants of decision attitude. 326 Journal of Behavioral Decision Making 7(2): 129-144.

327 Bell DE. 1982. Regret in decision making under uncertainty. Operations Research 30(5): 961-981.

328 Brassen S, Gamer M, Peters J, Gluth S, Büchel C. 2012. Don’t look back in anger! Responsiveness to 329 missed chances in successful and nonsuccessful aging. Science 336(6081): 612-614.

330 Büchel C, Brassen S, Yacubian J, Kalisch R, Sommer T. 2011. Ventral striatal signal changes represent 331 missed opportunities and predict future choice. Neuroimage 57(3): 1124-1130.

332 Camille N, Coricelli G, Sallet J, Pradat-Diehl P, Duhamel JR, Sirigu A. 2004. The involvement of the 333 orbitofrontal cortex in the experience of regret. Science 304(5674): 1167-1170.

334 Coricelli G, Dolan RJ, Sirigu A. 2007. Brain, emotion and decision making: the paradigmatic example 335 of regret. Trends in Cognitive Sciences 11(6): 258-265.

336 Coricelli G, Critchley HD, Joffily M, O'Doherty JP, Sirigu A, Dolan RJ. 2005. Regret and its 337 avoidance: A neuroimaging study of choice behavior. Nature Neuroscience 8(9): 1255-1262.

338 Creyer EH, Ross WT. 1999. The development and use of a regret experience measure to examine the 339 effects of outcome feedback on regret and subsequent choice. Marketing Letters 10(4): 373-386.

340 Fernandez-Duque D, Wifall T. 2007. Actor/observer asymmetry in risky decision making. Judgment 341 and Decision Making 2(1): 1-8. 
342 Finucane ML, Alhakami A, Slovic P, Johnson SM. 2000. The affect heuristic in judgments of risks and

343 benefits. Journal of Behavioral Decision Making 13(1): 1-17.

344 Hart S. 2005. Adaptive Heuristics. Econometrica 73(5): 1401-1430.

345 Hart S, Mas-Colell A. 2000. A simple adaptive procedure leading to correlated equilibrium.

346 Econometrica 68(5): 1127-1150.

347 Hoelzl E, Loewenstein G. 2005. Wearing out your shoes to prevent someone else from stepping into

348 them: Anticipated regret and social takeover in sequential decisions. Organizational Behavior and

349 Human Decision Processes 98(1): 15-27.

350 Janis IL, Mann L. 1977. Decision making: A psychological analysis of conflict, choice, and

351 commitment. New York: Free Press.

352 Kahneman D. 1995. Varieties of counterfactual thinking. In Roese NJ, Olson JM, eds. What might have

353 been: The social psychology of counterfactual thinking. Hillsdale: Lawrence Erlbaum Associates, Inc, $354 \quad 375-396$.

355 Kahneman D. 2003. A perspective on judgment and choice: Mapping bounded rationality. American

356 Psychologist 58(9): 697-720.

357 Kahneman D, Frederick S. 2002. Representativeness revisited: Attribute substitution in intuitive

358 judgment. In Gilovich T, Griffin D, Kahneman D, eds. Heuristics and biases: The psychology of

359 intuitive judgment. New York: Cambridge University Press, 49-81.

360 Keren GB, Wagenaar WA. 1985. On the psychology of playing blackjack: Normative and descriptive

361 considerations with implications for decision theory. Journal of Experimental Psychology: General

362 114(2): 133-158.

363 Larrick RP, Boles TL. 1995. Avoiding Regret in Decisions with Feedback: A Negotiation Example.

364 Organizational Behavior and Human Decision Processes 63(1): 87-97. 
365 Loomes G, Sugden R. 1982. Regret theory: an alternative theory of rational choice under uncertainty.

366 Economic Journal 92(368): 805-824.

367 Marcatto F, Ferrante D. 2008. The Regret and Disappointment Scale: An instrument for assessing

368 regret and disappointment in decision making. Judgment and Decision Making 3(1):87-99.

369 Marcatto F, Ferrante D. 2012. Regret in decision making. Italian Journal of Psychology 39(1):23-47.

370 Marchiori D, Warglien M. 2008. Predicting human interactive learning by regret-driven neural 371 networks. Science 319(5866):1111-1113.

372 Mellers BA, Schwartz A, Ritov I. 1999. Emotion-based choice. Journal of Experimental Psychology: 373 General 128(3):332-345.

374 O’Connor E, McCormack T, Feeney A. 2014. Do children who experience regret make better

375 decisions? A develpomental study of the behavioral consequences of regret. Child Development $376 \quad 85(5): 1995-2010$.

377 Raeva D, Mittone L, Schwarzbach J. 2010. Regret now, take it now: On the role of experienced regret 378 on intertemporal choice. Journal of Economic Psychology 31(4):634-642.

379 Raeva D, van Dijk E, Zeelenberg M. 2011. How comparing decision outcomes affects subsequent 380 decisions: The carry-over of a comparative mind-set. Judgment and Decision Making 6(4):343-350.

381 Ratner RK, Herbst,KC. 2005. When good decisions have bad outcomes: The impact of affect on 382 switching behavior. Organizational Behavior and Human Decision Processes 96(1):23-37.

383 Richard R, van der Pligt J, de Vries N. 1996. Anticipated regret and time perspective: Changing sexual 384 risk-taking behavior. Journal of Behavioral Decision Making 9(3):185-199.

385 Ritov I. 1996. Probability of regret: Anticipation of uncertainty resolution in choice. Organizational 386 Behavior and Human Decision Processes 66(2):228-236.

387 Saffrey C, Summerville A, Roese N. 2008. Praise for regret: People value regret above other negative 388 emotions. Motivation and Emotion 32(1):46-54. 
389 Simonson I. 1992. The influence of anticipating regret and responsibility on purchase decisions.

390 Journal of Consumer Research 19(1):105-118.

391 Slovic P. 1966. Risk-taking in children: Age and sex differences. Child Development 37(1):169-176.

392 van de Ven N, Zeelenberg M. 2011. Regret aversion and the reluctance to exchange lottery tickets.

393 Journal of Economic Psychology 32(1):194-200.

394 van Dijk E, Zeelenberg M. 2005. On the psychology of 'if only': Regret and the comparison between

395 factual and counterfactual outcomes. Organizational Behavior and Human Decision Processes

396 97(2):152-160.

397 van Dijk WW, Zeelenberg M. 2002. Investigating the appraisal patterns of regret and disappointment.

398 Motivation and Emotion 26(4):321-331.

399 Wright C, Ayton P. 2005. Focusing on what might happen and how it could feel: can the anticipation 400 of regret change students' computing-related choices?. International journal of human-computer 401 studies 62(6):759-783.

402 Zeelenberg M. 1999a. Anticipated regret, expected feedback and behavioral decision making. Journal 403 of Behavioral Decision Making 12(2):93-106.

404 Zeelenberg M. 1999b. The use of crying over spilled milk: a note on the rationality and functionality of 405 regret. Philosophical Psychology 12(3):325-340.

406 Zeelenberg M, Beattie J. 1997. Consequences of regret aversion 2: Additional evidence for effects of 407 feedback on decision making. Organizational Behavior and Human Decision Processes 72(1):63-78.

408 Zeelenberg M, Beattie J, van der Pligt J, de Vries NK. 1996. Consequences of regret aversion: Effects 409 of expected feedback on risky decision making. Organizational Behavior and Human Decision 410 Processes 65(2):148-158. 
411 Zeelenberg M, van Dijk WW, Manstead ASR, van der Pligt J. 2000. On bad decisions and

412 disconfirmed expectancies: The psychology of regret and disappointment. Cognition and Emotion

413 14(4):521-541.

414 Zeelenberg M, Pieters R. 2007. A theory of regret regulation 1.0. Journal of Consumer Psychology 415 17(1):3-18. 
Table $\mathbf{1}$ (on next page)

Mean emotional reaction ratings in the two experimental conditions.

Ratings ranged from 1 to 7 . 


\begin{tabular}{lcccc}
\hline & \multicolumn{2}{c}{ Regret condition } & \multicolumn{2}{c}{ Disappointment condition } \\
& Mean & SD & Mean & SD \\
\hline Negative affect & 3.16 & 1.55 & 3.09 & 1.58 \\
Regret score & 3.21 & 1.50 & 1.31 & 0.63 \\
Disappointment score & 3.68 & 1.45 & 5.23 & 1.50 \\
\hline
\end{tabular}

2 
Table 2 (on next page)

Mean and median of number of cards turned over in Red \& Black in each of the three conditions. 
2

\begin{tabular}{lccc}
\hline & Mean & SD & Median \\
\hline Control & 6.04 & 1.37 & 6 \\
Disappointment & 6.24 & 1.20 & 6 \\
Regret & 7.04 & 1.31 & 8 \\
\hline
\end{tabular}

3

4 